\title{
Gluconeogenesis and risk for fasting hyperglycemia in Black and White women
}

\author{
Stephanie T. Chung, ${ }^{1}$ Amber B. Courville, ${ }^{2}$ Anthony U. Onuzuruike, ${ }^{1}$ Mirella Galvan-De La Cruz, ${ }^{1}$ \\ Lilian S. Mabundo, ${ }^{1}$ Christopher W. DuBose, ${ }^{1}$ Kannan Kasturi, ${ }^{3}$ Hongyi Cai, ${ }^{1}$ Ahmed M. Charib, ${ }^{1}$ \\ Peter J. Walter, ${ }^{1}$ H. Martin Garraffo, ${ }^{1}$ Shaji Chacko, ${ }^{4}$ Morey W. Haymond, ${ }^{4}$ and Anne E. Sumner ${ }^{1,5}$ \\ ${ }^{1}$ National Institute of Diabetes and Digestive and Kidney Diseases (NIDDK), NIH, Bethesda, Maryland, USA. \\ ${ }^{2} \mathrm{NIH}$ Clinical Center (CC), Bethesda, Maryland, USA. ${ }^{3}$ Eunice Kennedy Shriver National Institute of Child Health and \\ Human Development, NIH, Bethesda, Maryland, USA. ${ }^{4}$ Department of Pediatrics, Children's Nutrition Research Center, US \\ Department of Agriculture/Agricultural Research Service, Baylor College of Medicine, Houston, Texas, USA. \\ ${ }^{5}$ National Institute of Minority Health and Health Disparities, NIH, Bethesda, Maryland, USA.
}

Black women, compared with White women, have high rates of whole-body insulin resistance but a lower prevalence of fasting hyperglycemia and hepatic steatosis. This dissociation of wholebody insulin resistance from fasting hyperglycemia may be explained by racial differences in gluconeogenesis, hepatic fat, or tissue-specific insulin sensitivity. Two groups of premenopausal federally employed women, without diabetes were studied. Using stable isotope tracers, $\left[{ }^{2} \mathrm{H}_{2} \mathrm{O}\right]$ and $\left[6,6^{2}-\mathrm{H}_{2}\right]$ glucose, basal glucose production was partitioned into its components (gluconeogenesis and glycogenolysis) and basal whole-body lipolysis ([ $\left.{ }^{2} \mathrm{H}_{5}\right]$ glycerol) was measured. Indices of insulin sensitivity, whole-body ( $\left.\mathrm{S}_{1}\right)$, hepatic ( $\mathrm{HISI}_{\mathrm{CPR}}$ ), and adipose tissue, were calculated. Hepatic fat was measured by proton magnetic resonance spectroscopy. Black women had less hepatic fat and lower fractional and absolute gluconeogenesis. Whole-body $\mathrm{S}_{1}, \mathrm{HISI}_{\mathrm{CPR}}$, and adipose tissue sensitivity were similar by race, but at any given level of whole-body $\mathrm{S}_{1}$, Black women had higher $\mathrm{HISI}_{\mathrm{CPR}}$. Therefore, fasting hyperglycemia may be a less common early pathological feature of prediabetes in Black women compared with White women, because gluconeogenesis remains lower despite similar whole-body $\mathrm{S}_{\text {. }}$

Conflict of interest: The authors have declared that no conflict of interest exists.

Submitted: April 5, 2018 Accepted: August 14, 2018 Published: September 20, 2018

\section{Reference information:} JCI Insight. 2018;3(18):e121495. https://doi.org/10.1172/jci. insight.121495.

\section{Introduction}

Black women experience disproportionately high rates of cardiometabolic disease and abnormal glucose tolerance (a term which encompasses both prediabetes and diabetes) $(1,2)$. As high rates of undiagnosed diabetes remain prevalent across populations of African ancestry, early risk recognition and effective implementation of prevention programs are paramount for reducing this chronic disease burden (3-5). Although there are multiple social and economic factors that contribute to these high undiagnosed rates, an important, underappreciated reason for these rates in populations of African ancestry may be the reduced sensitivity of fasting screening tests (3). Specifically, the fasting plasma glucose concentration (FPG) is a well-recognized screening tool $(6,7)$, but its diagnostic and predictive ability for abnormal glucose tolerance appears to vary by race (3). In the US, the prevalence of fasting hyperglycemia was approximately $30 \%-40 \%$ lower in Black adults compared with that in White adults, and the variance was not explained by differences in overall glycemia, age, sex, or BMI $(8,9)$. Similarly, if FPG was used alone to diagnose diabetes, the diagnostic sensitivity was only approximately $50 \%$ in US Black adults but $>85 \%$ when used in the entire adult cohort (10). This reduced diagnostic accuracy of FPG in diabetes has also been demonstrated in other Black populations, including African immigrants living in the US, Afro-Caribbeans, and native Africans living in sub-Saharan Africa (11-13).

The low prevalence rates of fasting hyperglycemia are also related to a low prevalence of 2 other traditional hepatic risk markers for diabetes (fasting hypertriglyceridemia and increased hepatic fat content) across Black populations around the world (14-20). The presence of these 3 risk factors predicts a faster decline in glucose tolerance, especially among individuals of European ancestry (21). Contrary to this paradigm, the relationship between these 3 traditional risk markers and whole-body insulin resistance is altered in Black populations - Black populations have high rates of whole-body insulin resistance and 
abnormal glucose tolerance but low or normal levels of fasting glucose, serum triglycerides, and hepatic fat (18). Moreover, these findings are observed across multiple populations of African ancestry (14-20), which suggests there maybe racial/ethnic variation in the development of fasting hyperglycemia and tissue-specific insulin resistance.

Yet, there is a relative absence of studies that have detailed the relationship between hepatic and peripheral insulin sensitivity, and the few results have contradicting conclusions (22-25). For example, two studies observed lower $(22,23)$ hepatic insulin sensitivity in Black women compared with White women, while one study identified higher (25) and another identified no differences (24) in insulin clamp measures of hepatic insulin sensitivity. Additionally, none of these analyses assessed hepatic relative to whole-body insulin sensitivity or hepatic risk markers. Therefore, at present, there is no consensus on why Black populations have lower prevalence rates of fasting hyperglycemia and lower hepatic fat but higher whole-body insulin resistance.

This study was designed to elucidate the mechanisms underlying the lower prevalence of fasting hyperglycemia and the relationship of hepatic to whole-body insulin sensitivity among Black and White women. During the normal postabsorptive state, glucose concentrations (70-99 $\mathrm{mg} / \mathrm{dl}$ ) are closely regulated by a network of neural, hormonal, and intracellular factors $(26,27)$. In the presence of risk factors, such as obesity, disruption of this neurohormonal network regulation causes fasting hyperglycemia and increased rates of both components of hepatic glucose production: (a) higher rates of gluconeogenesis; and (b) resistance to insulin-mediated suppression of glycogenolysis (28-30). We hypothesized that the lower prevalence of fasting hyperglycemia in Black women would be secondary to racial differences in the regulation of hepatic relative to whole-body insulin sensitivity and reflect pathophysiological distinctions in the development of increased hepatic glucose production. Our objectives were to compare by race rates of gluconeogenesis and glycogenolysis and to determine the relationship of hepatic to whole-body insulin sensitivity and hepatic fat in overweight/obese premenopausal Black and White women.

\section{Results}

Participant characteristics. Participant demographic and metabolic characteristics are depicted in Table 1. Black and White women were similar in age, and there were no group differences in BMI, waist circumference, total body fat, or lean body mass (LBM) (all $P>0.1$ ). Body fat distribution differed by group; Black women had significantly lower visceral and hepatic fat content $(P<0.05)$. The prevalence of prediabetes was similar by race: $17 \%$ Black ( $n=4$ of 24$)$ and $18 \%$ White $(n=4$ of 22$)$. Among the 8 women with prediabetes, there were no differences in age, BMI, waist circumference, or total body fat; however, hepatic fat content was lower in Black women compared with that in White women $(P<0.05$, Supplemental Table 1; supplemental material available online with this article; https://doi.org/10.1172/jci.insight.121495DS1). All 4 White women and only 1 of the 4 Black women with prediabetes had fasting hyperglycemia.

Basal hormone and substrate concentrations. Steady-state basal hormone concentrations were obtained after a 10- to 12 -hour overnight fast. Basal glucose $(92.1 \pm 1.5 \mathrm{mg} / \mathrm{dl} \mathrm{vs.} 93.0 \pm 1.2 \mathrm{mg} / \mathrm{dl})$, insulin $(11.6$ $\pm 1.5 \mu \mathrm{U} / \mathrm{ml}$ vs. $10.6 \pm 1.1 \mu \mathrm{U} / \mathrm{ml})$, C-peptide $(2.0 \pm 0.2 \mathrm{ng} / \mathrm{ml}$ vs. $2.4 \pm 0.2 \mathrm{ng} / \mathrm{ml})$, glucagon $(6.1 \pm$ $0.6 \mathrm{pmol} / 1$ vs. $6.3 \pm 0.6 \mathrm{pmol} / \mathrm{l})$, lactate $(258.0 \pm 15.4 \mu \mathrm{M}$ vs. $273.2 \pm 15.4 \mu \mathrm{M})$, and glycerol $(5.92 \pm$ $0.4 \mu \mathrm{g} / \mathrm{ml}$ vs. $6.64 \pm 0.3 \mu \mathrm{g} / \mathrm{ml}$ ) concentrations were similar in Black and White women (all $P>0.05$ ). The ratios of basal concentrations of insulin/glucagon and C-peptide/glucagon were not different by race (data not shown). Individual fasting fatty acid concentrations, measured by high-resolution liquid chromatography mass spectrometry $(\mu \mathrm{g} / \mathrm{ml})$, of myristic acid, myristoleic acid, palmitic acid, palmitoleic acid, and oleic acid were lower in Black versus White women (Figure 1, A-F, all $P \leq 0.01$ ), while total fasting free fatty acid (FFA) concentrations, measured by in vitro enzymatic colorimetric assay ( $\mathrm{mEq} / \mathrm{l}$ ), tended to be lower in Black versus White women (Figure $1 \mathrm{G}, P=0.09$ ). The stearoyl Co-A desaturase $\left(\mathrm{SCD}-1_{16}\right)$ index, an indicator of hepatic de novo lipogenesis, calculated as the plasma ratio of palmitoleic to palmitic acid, was also lower in Black versus White women (Figure $1 \mathrm{H}, P \leq 0.01$ ). There were no differences in concentrations of lauric, stearic, linoleic, arachidonic, eicosapentaenoic, or docosahexaenoic acids between the two groups $(P \geq 0.10)$.

Energy intake, energy expenditure, and substrate utilization. Total daily energy intake and macronutrient composition, obtained from 3- to 7-day food records, did not differ between the two groups of women (Supplemental Table 2). Similarly, total, sedentary, and moderate-to-vigorous habitual physical activity, objectively assessed by a 5- to 7-day accelerometer, were similar in both groups (Supplemental Table 3). 
Table 1. Participant characteristics

\begin{tabular}{|c|c|c|c|}
\hline Variable & Black $(n=24)$ & White $(n=22)$ & $P$ value \\
\hline Age (yr) & $37 \pm 1$ & $37 \pm 2$ & 0.93 \\
\hline $\mathrm{BMI}\left(\mathrm{kg} / \mathrm{m}^{2}\right)$ & $32.5 \pm 1.1$ & $32.4 \pm 1.0$ & 0.90 \\
\hline \multicolumn{4}{|l|}{ Body composition and fat distribution } \\
\hline Total fat mass $(\mathrm{kg})$ & $37.4 \pm 2.3$ & $38.6 \pm 1.8$ & 0.67 \\
\hline Total lean body mass $(\mathrm{kg})$ & $51.6 \pm 1.1$ & $50.7 \pm 1.5$ & 0.65 \\
\hline \multicolumn{4}{|l|}{ Metabolic characteristics } \\
\hline Prediabetes, $n(\%)$ & $4(17)$ & $4(18)$ & 0.89 \\
\hline Fasting hyperglycemia only, $n$ (\%) & $0(0)$ & $2(10)$ & 0.13 \\
\hline Impaired glucose tolerance only, $n$ (\%) & $3(13)$ & $2(10)$ & 0.89 \\
\hline Fasting glucose (mg/dl) & $90.8 \pm 1.3$ & $92.4 \pm 1.3$ & 0.39 \\
\hline \multicolumn{4}{|l|}{ Basal energy expenditure } \\
\hline REE (kcal·day $\left.{ }^{-1}\right)$ & $1,375 \pm 50$ & $1,511 \pm 39$ & 0.04 \\
\hline $\operatorname{REE}\left(\mathrm{kcal} \cdot \mathrm{kg}_{\text {LBM }}^{-1} \cdot \mathrm{day}^{-1}\right)$ & $28.5 \pm 1.0$ & $32.1 \pm 1.0$ & 0.01 \\
\hline RQ & $0.87 \pm 0.01$ & $0.83 \pm 0.01$ & $<0.01$ \\
\hline
\end{tabular}

Data are presented as mean \pm SEM, median $\left(25^{\text {th }}-75^{\text {th }}\right.$ percentile), or $n(\%) .{ }^{A}$ Natural log-transformed variable used. Groups were compared using unpaired Student's $t$ tests. REE, resting energy expenditure; RQ, respiratory quotient.

Indirect calorimetry was performed during the basal steady-state period of the overnight study. In Black women, resting energy expenditure (REE) was $136 \pm 63 \mathrm{kcal} \cdot \mathrm{day}^{-1}$ or $3.6 \mathrm{kcal} \cdot \mathrm{kg}^{-1}{ }_{\mathrm{LBM}} \cdot \mathrm{day}^{-1}$ lower and respiratory quotient (RQ) was $0.04 \pm 0.01$ higher compared with those of White women (Table $1, P<$ $0.05)$. In the 34 women with urinary nitrogen available, we calculated basal substrate oxidation rates. Black women had lower lipid oxidation rates $\left(138 \pm 6 \mathrm{vs} .159 \pm 8 \mathrm{mg} \cdot \mathrm{min}^{-1}, P=0.04\right)$ but no significant difference in glucose oxidation rates $\left(111 \pm 12 \mathrm{vs.} 90 \pm 9 \mathrm{mg} \cdot \mathrm{min}^{-1}, P=0.20\right)$.

Kinetic measurements. Using intravenous infusions of $\left[6,6-{ }^{2} \mathrm{H}_{2}\right]$ glucose, $\left[{ }^{2} \mathrm{H}_{5}\right]$ glycerol intravenous infusions, rates of glucose and glycerol turnover $(\mathrm{Ra})$ were calculated under near steady-state conditions ( -30 to 0 minutes). Fractional gluconeogenesis (the fraction of new glucose made overnight) was calculated using deuterated water $\left({ }^{2} \mathrm{H}_{2} \mathrm{O}\right)$ and the average deuterium technique (see Methods). The isotopic enrichments of $\left[6,6-{ }^{2} \mathrm{H}_{2}\right] \mathrm{glu}-$ cose, $\left[{ }^{2} \mathrm{H}_{5}\right]$ glycerol, average ${ }^{2} \mathrm{H}$ per glucose carbon and ${ }^{2} \mathrm{H}$ in body water under substrate, and isotopic steadystate conditions are given in Supplemental Table 4. Rates of fractional gluconeogenesis, glucose $\mathrm{Ra}$, and absolute gluconeogenesis were $9 \%$ lower in Black women (Figure 2, $P \leq 0.01$ ). Total rates of glucose production, the glucose Ra corrected for the tracer infusion rates, tended to be lower in Black compared with White women $\left(2.52 \pm 0.07\right.$ vs. $\left.2.71 \pm 0.06 \mathrm{mg} \cdot \mathrm{kg}_{\mathrm{LBM}}{ }^{-1} \cdot \mathrm{min}^{-1}, P=0.05\right)$. There were no significant differences in the rates of glycogenolysis or glycerol Ra (Figure $2 \mathrm{~B}$ ). The racial differences in glucose $\mathrm{Ra}$ and gluconeogenesis were observed when women without prediabetes were analyzed separately $(P<0.05$, data not shown). Similarly, in the 8 women with prediabetes, absolute rates of gluconeogenesis tended to be lower in Black women compared with White women $(P=0.08)$, but rates of glucose production, fractional gluconeogenesis, glycogenolysis, and glycerol Ra were similar $(P \geq 0.14$, Supplemental Table 1$)$.

Overall, fractional gluconeogenesis tended to or positively correlated with individual fatty acids, myristic acid, myristoleic acid, palmitic acid, palmitoleic acid, oleic acid, total FFA, and the SCD- $1_{16}$ index (all $r \geq 0.30$ ), but not with stearic acid (Figure $3, r=0.10$ ). There was no correlation between fractional gluconeogenesis and whole-body $\mathrm{S}_{\mathrm{I}}$, glycerol concentrations, glycerol Ra, liver fat, or visceral fat (all $\mathrm{r}=0.10, P$ $>0.05)$. Rates of glucose production positively correlated with REE ( $\mathrm{r}=0.40, P<0.01)$ but not with RQ (r $=0.16, P<0.48)$, rates of lipid oxidation $(\mathrm{r}=-0.10, P=0.61)$, or steady-state fasting glucose $(\mathrm{r}=0.14, P$ $=0.33$ ), and these relationships did not differ by race. To determine if the lower REE may have contributed 
A

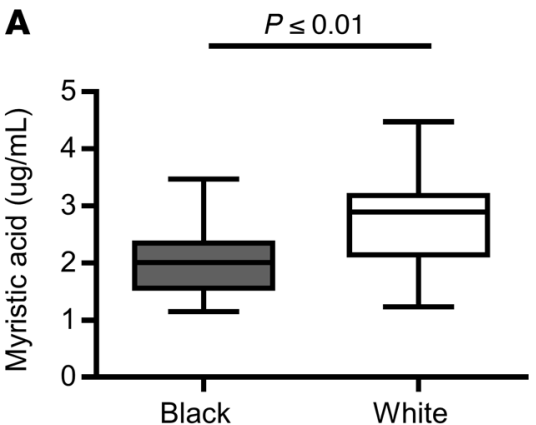

C
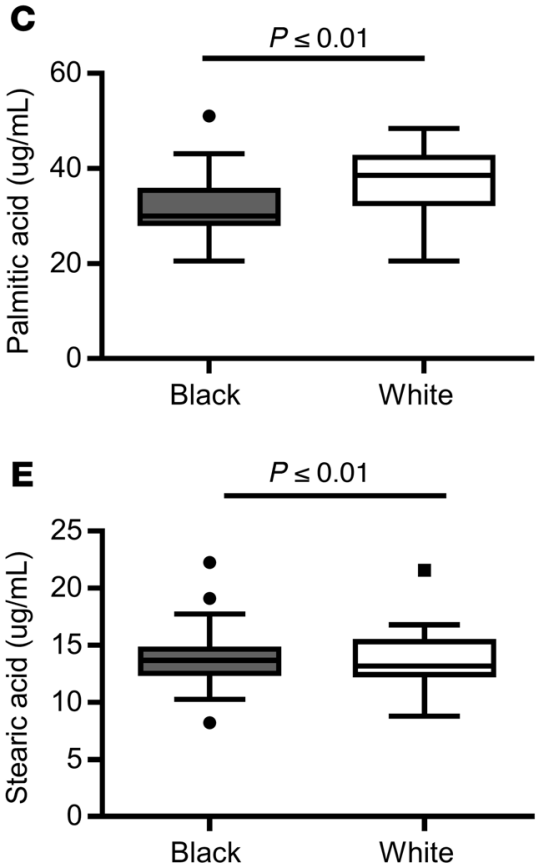

\section{G}

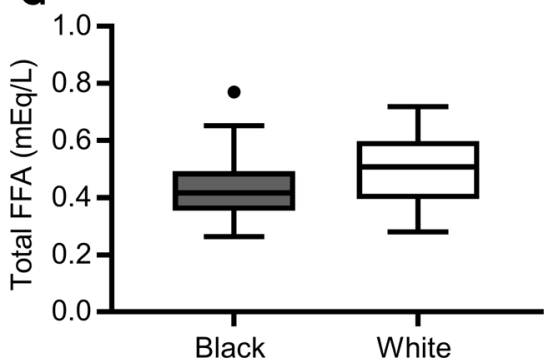

B

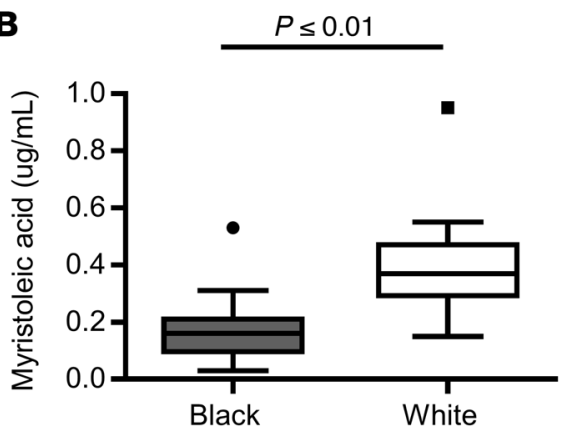

D

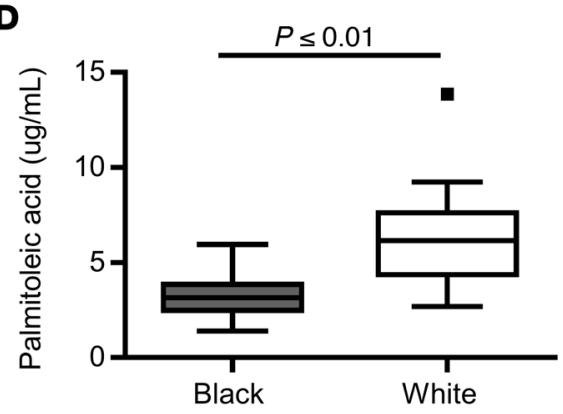

$\mathbf{F}$

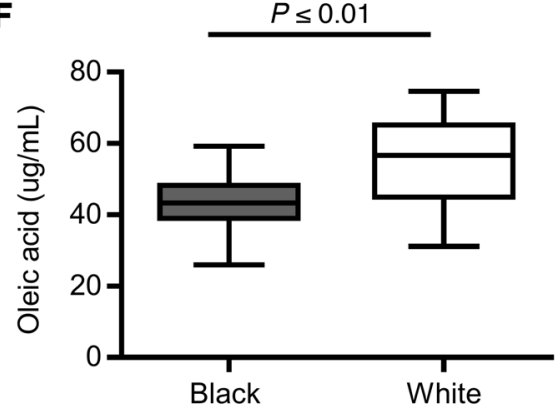

H

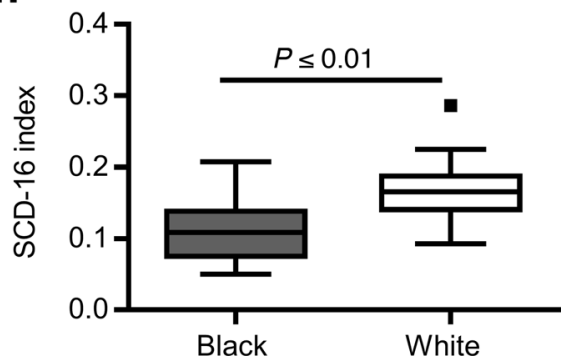

Figure 1. Individual fatty acid concentrations and stearoyl Co-A desaturase activity by race. Tukey box-and-whisker plots of (A) myristic acid, (B) myristoleic acid, (C) palmitic acid, (D) palmitoleic acid, (E) stearic acid, (F) oleic acid, (G) total free fatty acid (FFA), and (H) SCD-1 ${ }_{16}$ index in Black (gray, $n=24$ ) and White (white, $n=22$ ) women. Top and bottom box limits represent the 75th and 25th percentile, respectively; midlines represent the median; and top and bottom whiskers represent the 75 th percentile plus 1.5 of the interquartile range (IQR) and the 25 th percentile minus $1.5 \mathrm{IQR}$, respectively. Black dots represent any values that lie outside the range of the whiskers. Individual fatty acid concentrations were measured by high-resolution liquid chromatography mass spectrometry. The stearoyl Co-A desaturase 1 activity $\left(S C D-1_{16}\right)$ index is an indicator of hepatic de novo lipogenesis, calculated as the plasma ratio of palmitoleic to palmitic acid. Groups were compared by unpaired Student's $t$ test.

to the differences in gluconeogenesis observed, we adjusted the rates of glucose production and gluconeogenesis for the REE and the results did not change (data not shown).

Insulin sensitivity and response. Table 2 illustrates the comparison of whole-body, hepatic, and adipose tissue insulin sensitivity indices by race. Whole-body $\mathrm{S}_{\mathrm{I}}$ was calculated during an unlabeled insulin-modified frequently sampled intravenous glucose tolerance test (IM-FSIGT); adipose tissue insulin sensitivity was calculated as the product of glycerol Ra and steady-state insulin concentrations; and hepatic insulin sensitivity $\left(\mathrm{HISI}_{\mathrm{GPR}}\right.$ ) was calculated as the product of glucose production rate and steady-state insulin concentrations. Using unpaired $t$ tests to compare means in Black and White women, whole-body $\mathrm{S}_{\mathrm{I}}, \mathrm{HISI}_{\mathrm{GPR}}$, and adipose tissue insulin sensitivity indices were not different (Table 2). To determine the relationship between HISI $_{\text {GPR }}$ and whole-body $\mathrm{S}_{\mathrm{I}}$, we used a linear regression model with race as a covariate. Black women had higher $\mathrm{HISI}_{\mathrm{GPR}}$ at any given level of whole-body $\mathrm{S}_{\mathrm{I}}$ compared with White women ( $\beta=11.8 ; 95 \% \mathrm{CI}$ : 3.1, 
A

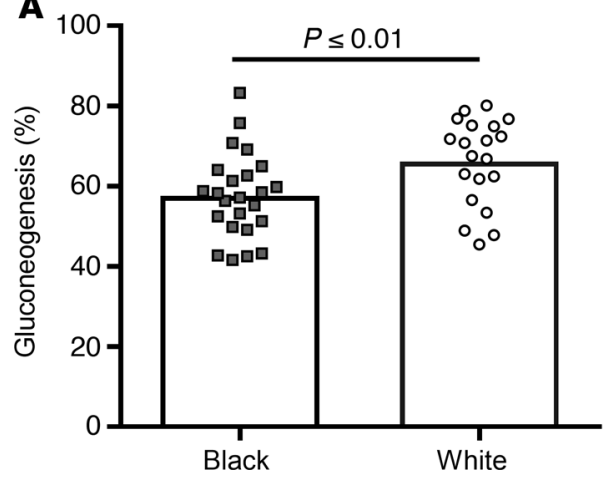

B

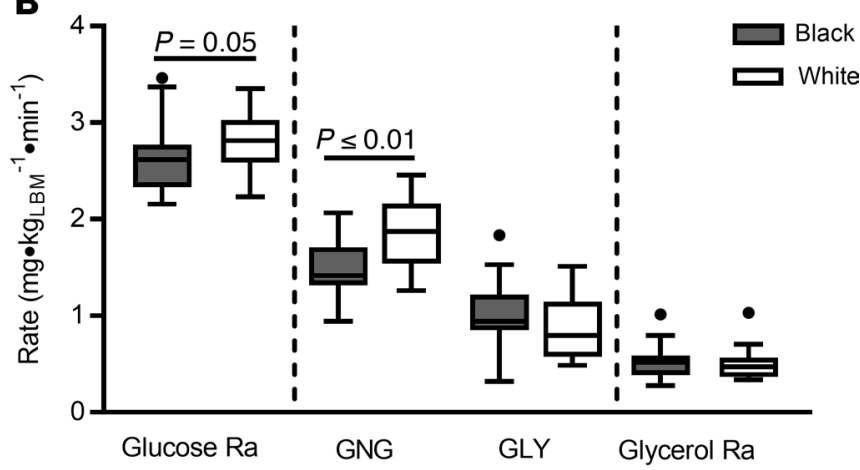

Figure 2. Basal rates of glucose and glycerol turnover in Black and White women. Using stable isotope tracers after a 10- to 12-hour overnight fast, we compared basal rates of (A) fractional gluconeogenesis (GNG) (boxes represent mean per group, with individual data points in Black women [gray squares, $n=24$ ] and White women [white circles, $n=22$ ]) and (B) glucose turnover (Ra), absolute GNG, glycogenolysis (GLY), and glycerol Ra (Tukey box plots) in Black women (gray, $n=24$ ) and White women (White, $n=22$ ). Top and bottom box limits represent the 75th and 25th percentile, respectively; midlines represent the median; and top and bottom whiskers represent the 75 th percentile plus 1.5 of the interquartile range (IQR) and the 25th percentile minus 1.5 IQR, respectively. Black dots represent any values that lie outside the range of the whiskers. Groups were compared by unpaired Student's $t$ test. LBM, lean body mass.

20.4; adjusted $\mathrm{R}^{2}=0.64 ; P<0.01$; Figure $\left.4 \mathrm{~A}\right)$. There was no interaction between whole-body SI and race $(P>0.50)$. HISI $_{\mathrm{GPR}}$ negatively correlated with hepatic risk markers, hepatic fat (Figure $\left.4 \mathrm{~B}\right)$ as well as visceral fat, and fasting triglyceride concentrations, with no difference in these associations by race (Table 3 ).

During the IM-FSIGT, two measures of insulin response were derived: the acute insulin response to glucose (AIRg) and the disposition index (DI) calculated as AIRg $\times \mathrm{S}_{\mathrm{I}}$. Black women compared with White women had higher AIRg (715 [range, 577-1,430] $\mathrm{mU} \cdot 1^{-1} \cdot \mathrm{min}^{-1}$ vs. 540 [range, 395-722] $\mathrm{mU} \cdot 1^{-1} \cdot \mathrm{min}^{-1}$, median [25th-75th percentile], $P=0.02)$ and DI (1,776 [range, 1,241-2,354] vs. 1,438 [range, 991-1,817], $P=0.04)$.

\section{Discussion}

This study provides insight into racial differences in the prevalence of fasting hyperglycemia by comparing the components of hepatic glucose production (gluconeogenesis and glycogenolysis) and the relationship of hepatic insulin sensitivity to whole-body $\mathrm{S}_{\mathrm{I}}$ in Black and White premenopausal women. We demonstrated that Black women had lower basal rates of gluconeogenesis compared with White women, despite similar whole-body $\mathrm{S}_{\mathrm{I}}$ (Figure 2). Since rates of glycogenolysis were comparable between the two groups, lower glucose production was secondary to lower fractional and absolute rates of gluconeogenesis. Moreover, approximately $10 \%$ lower rates of gluconeogenesis were observed, even though basal insulin concentrations and the incidence of prediabetes were similar between Black and White women.

These observations support our hypothesis that there are racial differences in gluconeogenesis and have important pathophysiological implications. Lower rates of gluconeogenesis observed in Black women were associated with normal fasting glucose concentrations, despite a similar prevalence of prediabetes and impaired glucose tolerance. Thus, increased gluconeogenesis, a well-established early pathological feature of diabetes, was uncommon in Black women who were overweight or obese, elucidating a potential mechanism to explain why FPG is a poor early marker of prediabetes in populations of African ancestry (11-13). While these findings could help to explain the lower prevalence of fasting hyperglycemia observed in epidemiological studies of populations of African ancestry $(8,9)$, our cohort of women with prediabetes was small and larger studies are needed for confirmation.

Past analyses that have attempted to explain the lower prevalence of fasting hyperglycemia in Black individuals have not examined the components of hepatic glucose production or its relationship with hepatic and whole-body insulin sensitivity (22-25). Our findings of lower basal rates of gluconeogenesis and higher $\mathrm{HISI}_{\mathrm{GPR}}$ at any given whole-body $\mathrm{S}_{\mathrm{I}}$ contrast with findings of two previous studies that identified lower hepatic insulin sensitivity in Black women compared with White women $(22,23)$. However, these two studies were limited by between-group heterogeneity in basal insulin concentrations, while, in the current study, the comparisons by race were not conflated by differences in body composition or insulin resistance. Moreover, our results of higher HISI $_{\mathrm{GPR}}$ for a given whole-body $\mathrm{S}_{\mathrm{I}}$ are in agreement with two other 

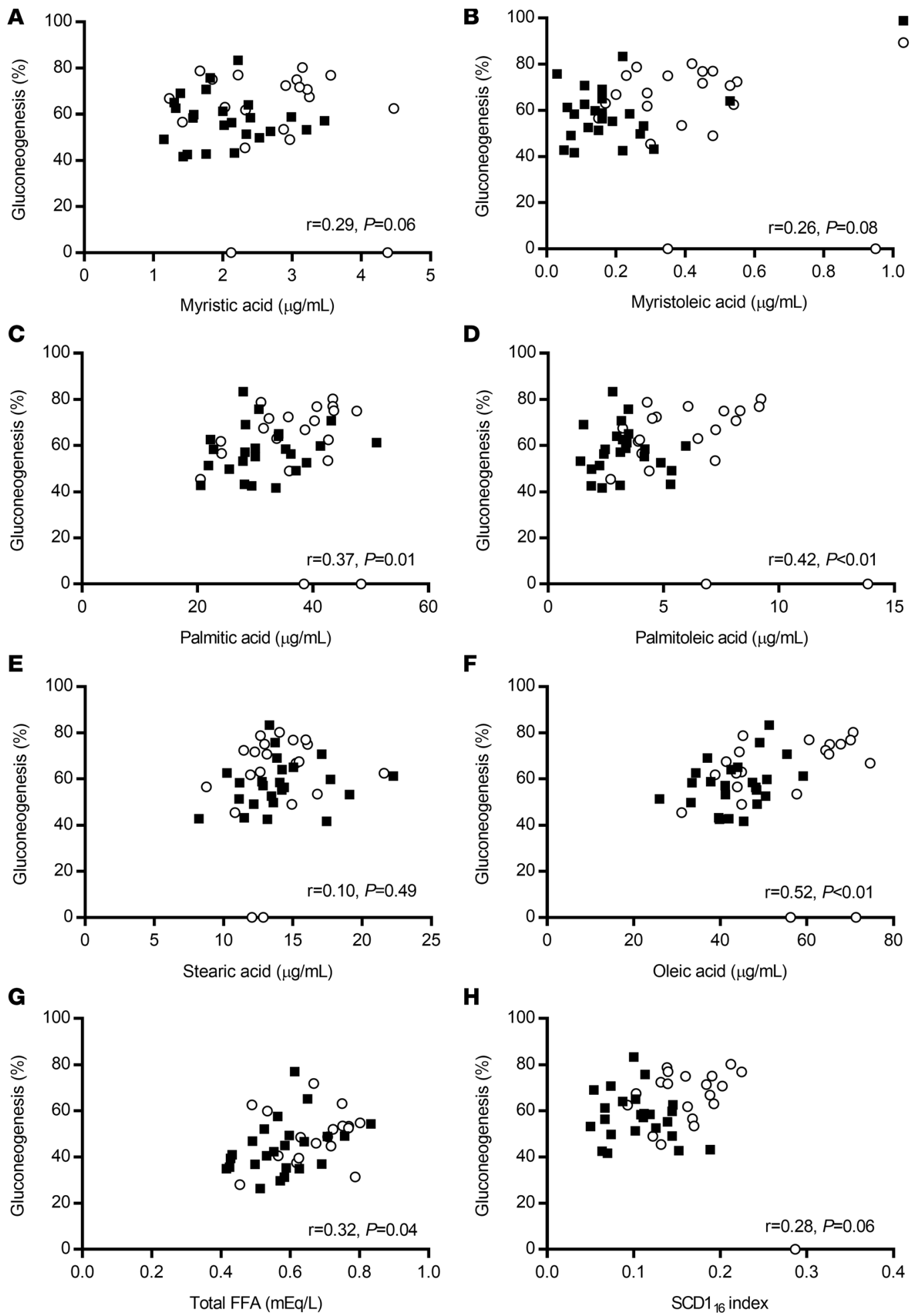

Figure 3. The relationship of fractional gluconeogenesis with fatty acid concentrations and stearoyl Co-A desaturase activity. Scatterplots and Pearson correlations for (A) myristic acid, (B) myristoleic acid, (C) palmitic acid, (D) palmitoleic acid, (E) stearic acid, (F) oleic acid, (G) total free fatty acid (FFA), and (H) SCD-1 $1_{15}$ in Black women (black box and solid line, $n=24$ ) and White women (white circle, dotted line, $n=22$ ). SCD-1 $1_{16}$, stearoyl Co-A desaturase 1 activity index. SCD-1 $1_{16}$ is an indicator of hepatic de novo lipogenesis, calculated as the plasma ratio of palmitoleic to palmitic acid. 
Table 2. Indices of insulin sensitivity

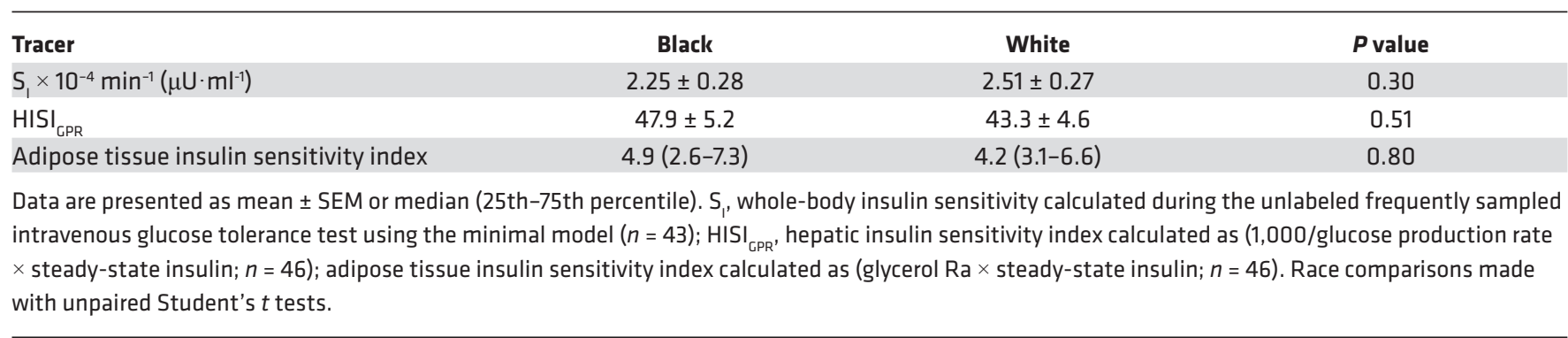

studies that used an independent measure of hepatic insulin sensitivity, suppression of hepatic glucose production during a controlled hyper insulinemic euglycemic clamp, and found higher or no differences in hepatic insulin sensitivity but lower peripheral (muscle) insulin sensitivity in women $(24,25)$. To further elucidate this relationship between tissue-specific and whole-body insulin sensitivity by race, we also calculated adipose tissue insulin sensitivity during the basal steady-state period. Both hepatic and adipose tissue indices positively correlated with whole-body $\mathrm{S}_{\mathrm{I}}$, regardless of race, but only $\mathrm{HISI}_{\mathrm{GPR}}$ was higher in Black women compared with White women, at every level of whole-body $\mathrm{S}_{\mathrm{I}}$ (Figure 4A).

The finding that HISI $_{\text {GPR }}$ was higher at a given level of whole-body $\mathrm{S}_{\mathrm{I}}$ in Black women also helps to explain the "insulin resistance paradox," an epidemiological association describing high rates of whole-body insulin resistance but a low prevalence of hepatic steatosis and hypertriglyceridemia in Black individuals (14). This paradoxical relationship is characterized by relatively greater subcutaneous fat accretion compared with visceral and hepatic adipose tissue deposition (14-20). We now extend the literature by showing that the metabolic phenotype of lower visceral and hepatic fat content in Black women was also associated with lower rates of gluconeogenesis and relatively higher $\mathrm{HISI}_{\mathrm{GPR}}$. Additionally, traditional diabetes risk markers inversely correlated with HISI $_{\mathrm{GPR}}$ regardless of race (Table 3), indicating that the lower hepatic and visceral fat and fasting triglycerides do reflect improved hepatic insulin sensitivity in Black women.

Contrary to our results, hepatic insulin sensitivity is presumed to be lower in Black individuals because high diabetes rates and lower whole-body $\mathrm{S}_{\mathrm{I}}$ is often associated with lower insulin clearance (31-34). Indeed, a recent mathematical model of C-peptide concentrations during an IM-FSIGT found differential regulation in insulin clearance in hepatic and extra-hepatic tissues (35), and lower hepatic insulin clearance in Black compared with White individuals (36). However, to our knowledge, a causal association between hepatic insulin clearance and hepatic insulin sensitivity in Black individuals has not been fully explored. In the larger group of the Federal Women's Study, lower insulin clearance was similarly associated with lower hepatic fat content (Chung et al., unpublished observations). We propose that intrinsic racial differences in insulin degradation pathways could explain lower insulin clearance but higher hepatic insulin sensitivity in Black individuals (37). Although we did not compute hepatic insulin clearance in this study, basal insulin concentrations were similar in the 2 groups, implying that insulin clearance was not different under fasting and steady-state isotopic conditions. Therefore, at least in the basal period, higher hepatic insulin sensitivity at a given level of $\mathrm{S}_{\mathrm{I}}$ in Black women appears to be independent of racial differences in insulin clearance.

Another important physiologic implication of this study is that differential regulation of gluconeogenic pathways may occur before the development of prediabetes because lower rates of gluconeogenesis were also observed in women without prediabetes. Notably, the lower prevalence of fasting hyperglycemia in Black individuals is not explained by a population shift in the distribution of FPG. In nondiabetic individuals, there is minimal variation reported in the distribution of FPG in non-Hispanic Black individuals in the US, and FPG was only 1-3 mg/dl lower than in White and Hispanic individuals (38). Data across the African diaspora are limited, but FPG is also comparable among Black individuals living in US, Jamaica, Seychelles, and Ghana (39).

To further understand why gluconeogenesis would be differentially regulated by race, we examined its relationship with hormonal and substrate concentrations. Basal ratios of insulin/glucagon and C-peptide/glucagon concentrations were similar, and insulin's ability to suppress rates of glycogenolysis, the component of hepatic glucose production that is most sensitive to insulin's suppressive effect (40), did 

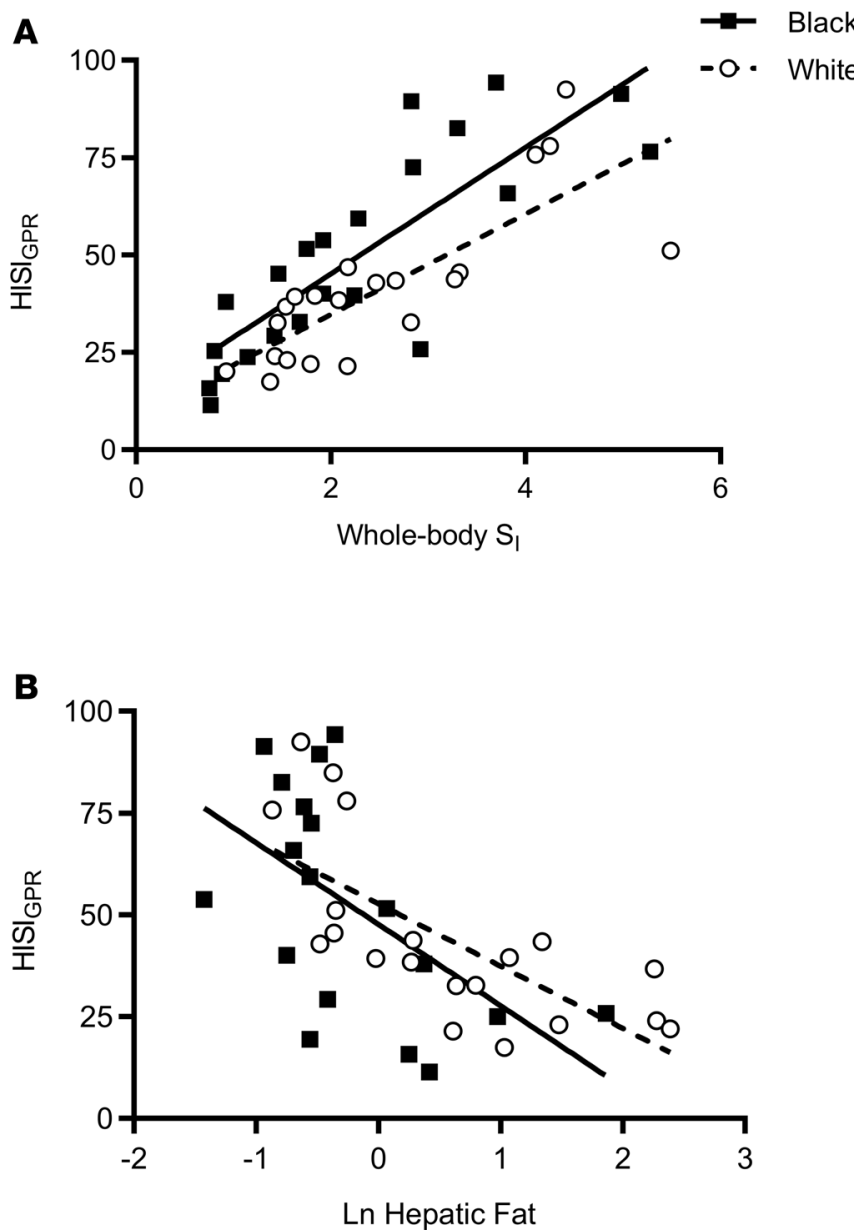

Figure 4. Relationship of hepatic insulin sensitivity index with whole-body insulin sensitivity and hepatic fat. (A) Scatter plot of hepatic insulin sensitivity index ( $\mathrm{HISI}_{\mathrm{CPR}}$ ) with whole-body insulin sensitivity $\left(\mathrm{S}_{1}\right)$ in Black women (black squares and solid line, $n=22$ ) and White women (white circles and dotted line, $n=21$ ). Using linear regression analyses, Black women had higher HISI ${ }_{\mathrm{G}-}$ ${ }_{P R}$ at any given level of whole-body $S_{1}(\beta$ $=11.8 ; 95 \% \mathrm{Cl}$ : 3.1, 20.4; adjusted $\mathrm{R}^{2}=$ $0.64 ; P<0.01)$. There was no interaction between whole-body $\mathrm{S}$, and race $(P>$ 0.50). (B) Scatter plot of HISI $\mathrm{CPR}_{\text {with }}$ Ln hepatic fat in Black women (black squares and line, $n=18$ ) and White women (White circles and dotted line, $n$ $=20)$. Ln, natural log-transformed. Pearson correlations were used to determine correlation coefficients (black, $r=-0.6$, and White, $r=-0.7, P \leq 0.01$ ).

not differ by race. Therefore, nonhormonal factors, including mediators other than differences in ambient insulin concentrations and insulin clearance, may play a primary role. Instead, racial differences in macronutrient intake and substrate oxidation could alter rates of gluconeogenesis by changing substrate availability and flux through the tricarboxylic acid cyclic (41). We evaluated and found no racial differences in daily energy intake (Supplemental Table 2), daily energy expenditure (Supplemental Table 3), or energy balance (assessed by weight, Table 1). In addition, each participant received a standardized dinner meal the evening prior to the overnight fast that consisted of similar macronutrient and energy composition (Black, $9 \pm 0.3 \mathrm{kcal} / \mathrm{kg}$, and White, $9.2 \pm 0.2 \mathrm{kcal} / \mathrm{kg}, P=0.5$ ). Further, no differences in two key gluconeogenic substrates (lactate or glycerol concentrations) were noted between the groups. While not a gluconeogenic substrate, total FFAs tended to be lower and individual fatty acids were lower in Black women and correlated with gluconeogenesis (Figures 2 and 3). FFAs do not provide direct carbons for gluconeogenesis, but their hepatic oxidation provides the energy needed to drive gluconeogenesis, and lower FFA release from hepatic fat could reduce energy needed to drive the gluconeogenic process (42). In the setting of weight stability and similar macronutrient intake, the lower fatty acid concentrations in Black women could result from decreased FFA release or increased FFA clearance. Rates of gluconeogenesis correlated with a marker of hepatic de novo lipogenesis, SCD-1 ${ }_{16}$, indicating that lower hepatic stores may contribute, at least in part, to the differences in gluconeogenesis between these two groups of women. Alternatively, greater FFA clearance in Black individuals may be a consequence of relative hyperinsulinemia (as noted in this study) and has been demonstrated previously (43-47). Overall, regardless of the source (FFA release or clearance), lower fatty acid concentrations correlated with gluconeogenesis and lower basal REE. However, there was no correlation of lipid oxidation with gluconeogenesis, and when rates of gluconeogenesis were adjusted for REE, rates of gluconeogenesis were still 10\% lower in Black women. Additional studies are required to determine whether variations in fatty acid availability and/or kinetics could explain the lower basal rates of gluconeogenesis observed in Black women. 
Table 3. Relationship of hepatic insulin sensitivity with traditional diabetes risk markers

\begin{tabular}{|c|c|c|c|c|}
\hline \multirow[b]{2}{*}{$\mathrm{HISI}_{\mathrm{GPR}}$} & \multicolumn{2}{|c|}{ Black } & \multicolumn{2}{|c|}{ White } \\
\hline & Correlation (r) & $P$ value & Correlation (r) & $P$ value \\
\hline Visceral fat & -0.77 & $<0.01$ & -0.67 & 0.02 \\
\hline Hepatic fat & -0.56 & 0.02 & -0.70 & $<0.01$ \\
\hline $\begin{array}{l}\text { Fasting triglyceride } \\
\text { concentration }\end{array}$ & -0.46 & 0.02 & -0.43 & 0.01 \\
\hline \multicolumn{5}{|c|}{$\begin{array}{l}\text { Correlation coefficients }(r) \text { obtained with Pearson correlations of HISI }{ }_{\text {GPR }} \text { with visceral and hepatic fat }(B l a c k, n=18 \text { and White, } n=17) \text { and fasting } \\
\text { triglyceride (Black, } n=24 \text { and White, } n=22 \text { ). HISI } \text { GPR }_{\text {, hepatic sensitivity index. Visceral fat, hepatic fat, and fasting triglyceride concentrations were natural }} \\
\text { log-transformed for the Pearson correlational analyses. }\end{array}$} \\
\hline
\end{tabular}

Study strengths and limitations. Our study has several strengths. First, we recruited federally employed premenopausal women to ensure that they all had health insurance and access to health care; both groups of women were similar in age, BMI, daily energy intake, and physical activity levels. Since both groups had comparable whole-body $\mathrm{S}_{\mathrm{I}}$, this enabled direct comparisons of substrate kinetics and tissue-specific insulin sensitivity. Second, our postabsorptive study design, measuring the components of hepatic glucose production, allowed determination of two pathways that contribute to fasting hyperglycemia: the insulin-sensitive pathway, in which rates of glycogenolysis are most sensitive to insulin (40), and alternative regulators of increased gluconeogenesis, such as substrate availability. However, a few limitations are noteworthy. As this was a cross-sectional analysis in premenopausal women, we cannot infer whether tissue-specific insulin sensitivity will change over time in response to age or rapid weight gain or if these findings are generalizable to men or postmenopausal women. It is also unclear whether these racial differences in gluconeogenesis or hepatic insulin sensitivity would persist under glucose- and insulin-stimulated conditions, such as during a meal. Finally, this cross-sectional analysis compares metabolically healthy Black and White women who were overweight or obese, and larger studies of men and women with prediabetes are needed to determine whether the relatively small differences in glucose production observed in this cohort directly contribute to the lower prevalence of fasting hyperglycemia in the larger population.

Conclusion. In conclusion, type 2 diabetes is a leading cause of death and disability in Black populations worldwide, but fasting hyperglycemia and hepatic steatosis are uncommon early pathological features of their abnormal glucose tolerant state. By demonstrating that gluconeogenesis was lower in Black women, compared with White women who were overweight or obese and had similar whole-body $\mathrm{S}_{\mathrm{I}}$, we have elucidated, at least in part, racial differences in the physiological mechanisms that may contribute to the development of fasting hyperglycemia. The lower rates of gluconeogenesis in Black women may explain why using FPG alone could contribute to lower risk discrimination in diabetes prediction models and missed opportunities for early intervention $(8,48)$. Alternative and combination strategies, such as combining FPG with $\mathrm{HbA1c}$ tests (13), may be useful for improving primary and secondary prevention programs in individuals of African ancestry.

\section{Methods}

Study population and design. Sixty-eight participants were recruited and screened from newspaper advertisements, flyers, or the NIH website. Participants were enrolled in a cross-sectional study to evaluate cardiometabolic health in African immigrant, Black, and White women who were employed by the federal government (the Federal Women's Study, Clinicaltrials.gov NCT01809288). Of the 68 participants, 46 women met all inclusion criteria (Supplemental Figure 1): self-identified as healthy and were 25-50 years of age, overweight or obese (BMI $\geq 25 \mathrm{~kg} / \mathrm{m}^{2}$ ), and premenopausal ( $\mathrm{FSH}<22 \mathrm{U} / 1$ ). Black women of African ancestry were defined as Black (parents and participant identified as Black born in the US) or African (parents and participant born in Africa). White women identified themselves and both parents as White.

The study protocol was conducted at the NIH CC and consisted of 3 visits. At visit 1 (screening), a history and physical examination were performed, and laboratory studies were done to exclude the presence of anemia, diabetes, liver, or kidney disease. Participants on nutritional supplements or medications known to affect triglyceride concentrations or insulin sensitivity (including oral contraceptive pills) were excluded. Visits 2 and 3 were at least 1 week apart and completed within a 6-week time frame. At visit 2, women came 
to the NIH CC Day Hospital after a 10- to 12-hour overnight fast and underwent a standard 75-g oral glucose tolerance test and dual-energy X-ray absorptiometry (DXA) scan for determination of fat and fat free mass. At visit 3 (overnight tracer study), women were admitted to the Metabolic Research Unit at the NIH CC the afternoon before the study day. Women received a standardized dinner meal (45\% carbohydrate, $35 \%$ fat, and $20 \%$ protein) at 1800 hours, which provided approximately $35 \%$ of the participants' estimated energy needs (calculated using the Mifflin St. Joer equation plus an activity factor of 1.5) (49). Overnight, participants had ad libitum access to water and were fasted from 2100 hours on the night of admission until the termination of the inpatient study at approximately 1200 hours the following day. A 12-hour urine collection was initiated at $6 \mathrm{PM}$ on the day of admission and lasted until 6 AM on the following day.

Tracer administration and assessment of insulin sensitivity. Participants received $3.0 \mathrm{~g} \cdot \mathrm{kg}_{\mathrm{LBM}}{ }^{-1}$ of deuterium oxide $\left[{ }^{2} \mathrm{H}_{2} \mathrm{O}\right]$ orally, given in 3 divided doses every 3 hours (2100 hours, 2400 hours, and 0300 hours) to enrich the body water pool to approximately $0.3 \%{ }^{2} \mathrm{H}_{2} \mathrm{O}$ to measure fractional gluconeogenesis. At 0400 hours the following morning, a simultaneous, primed $(60 \times$ the minute infusion rate), 5-hour constant rate infusion was initiated with $\left[6,6{ }^{2} \mathrm{H}_{2}\right]$ glucose $\left(0.09 \mathrm{mg} \cdot \mathrm{kg}_{\mathrm{LBM}}{ }^{-1} \cdot \mathrm{min}^{-1}\right)$ and $\left[{ }^{2} \mathrm{H}_{5}\right]$ glycerol $(0.015 \mathrm{mg} \cdot \mathrm{k}-$ $\mathrm{g}_{\mathrm{LBM}}{ }^{-1} \cdot \mathrm{min}^{-1}$ ) to measure rates of production of glucose and glycerol, respectively. Between 0830 and 0900 hours, blood samples were obtained at $-30,-20,-10$, and 0 minutes to assess plasma glucose, insulin, and $\mathrm{C}$-peptide concentrations and isotopic enrichments during the near-steady state period. Isotope infusions were subsequently discontinued and an unlabeled IM-FSIGT was performed, with plasma glucose, insulin, and C-peptide concentrations obtained at 32 time points between baseline and 180 minutes $(50,51)$.

Energy intake, expenditure, and physical activity. Three- to seven-day food records were collected, reviewed, and analyzed by nutrition staff to assess daily energy and macronutrient intake. Habitual physical activity and sedentary time were assessed with an accelerometer (Actigraph) worn for 5-7 days in between visits 2 and 3. At visit 3 (overnight tracer), REE was assessed via indirect calorimetry using the ParvoMedics TrueOne 2400 Metabolic Measurement System. Measurements of oxygen consumption and carbon dioxide production via indirect calorimetry were performed during steady state on study day 2 at between 0700 and 0730 hours. Due to technical difficulties, REE was not available in 2 Black women. Substrate oxidation rates were computed using 12-hour excretion of urinary nitrogen $(52,53)$ in 20 Black and 14 White women.

Imaging studies. Whole-body composition measurements were performed with DXA scans (Hologic Discovery). Measurements of liver and visceral fat content were performed at L2-3 level by magnetic resonance spectroscopy as previously described (54).

Tracers. Deuterium oxide $\left(99 \%\left[{ }^{2} \mathrm{H}\right]\right),\left[6,6-{ }^{2} \mathrm{H}_{2}\right]$ glucose $\left(99 \%\left[{ }^{2} \mathrm{H}\right]\right)$, and $\left[{ }^{2} \mathrm{H}_{5}\right]$ glycerol $\left(99 \%\left[{ }^{2} \mathrm{H}\right]\right)$ were purchased from Cambridge Isotope Laboratories. The isotopes were prepared and tested for sterility and pyrogenicity by the Pharmaceutical Development Service at the NIH CC and Pine Pharmaceuticals LLC.

Analyses. Glucose concentrations were measured in sera using an enzymatic hexokinase assay on the Cobas 6000 instrument (Roche Diagnostics). Insulin and C-peptide were measured in sera via electrochemiluminescence on the Cobas 6000 instrument (Roche Diagnostics). Hemoglobin A1c was determined by the HPLC D10 instrument (Bio-Rad). Plasma and urine samples for the analysis of FFAs, glucagon, lactate, and nitrogen were stored at $-80^{\circ} \mathrm{C}$ and then processed and analyzed simultaneously. Total FFAs were measured by an in vitro enzymatic colorimetric assay (Wako diagnostics), L-lactate was measured by a colorimetric fluorescence assay (Cayman Chemical Company), and glucagon was measured by a solid-phase two-site enzyme immunoassay (Mercodia). Urinary nitrogen concentrations were measured by a Antek MultiTek Horizontal nitrogen analyzer. Plasma glycerol and individual fatty acid concentrations were measured by liquid chromatography mass spectrometry (NIDDK) (55). The enrichments of $\left[{ }^{2} \mathrm{H}_{2} \mathrm{O}\right]$ water, $\left[{ }^{2} \mathrm{H}_{1}\right]$ glucose, $\left[{ }^{2} \mathrm{H}_{5}\right]$ glycerol, and $\left[{ }^{2} \mathrm{H}_{2}\right]$ glucose were determined as previously described $(56,57)$.

Calculations and definitions. Prediabetes was defined using American Diabetes Association criteria: FPG (fasting glucose $\geq 100 \mathrm{mg} / \mathrm{dl}$ ) and/or impaired glucose tolerance (2-hour glucose $\geq 140 \mathrm{mg} / \mathrm{dl}$ ). Rates of plasma glucose appearance and glycerol production were calculated under near steady-state conditions ( -30 to 0 minutes) using the average enrichment of $\left[6,6{ }^{2} \mathrm{H}_{2}\right]$ glucose and $\left[{ }^{2} \mathrm{H}_{5}\right]$ glycerol, with conventional isotope dilution calculations (58). Under fasting and both isotopic and substrate steady-state conditions, when corrected for the rate of infusion of the tracer, the rate of glucose appearance $(\mathrm{Ra})$ is the glucose production. Fractional gluconeogenesis was determined using ${ }^{2} \mathrm{H}_{2} \mathrm{O}$ and the average enrichment of ${ }^{2} \mathrm{H}$ enrichments of carbons $1,3,4,5,6$ of glucose as previously described (56). Since body water is the precursor pool for the hydrogen incorporated into glucose during gluconeogenesis, the extent of deuterium labeling of glucose during the gluconeogenic 
process is a measure of fractional gluconeogenesis. The rate of gluconeogenesis was calculated as the product of glucose $\mathrm{Ra}$ and fractional gluconeogenesis (glucose $\mathrm{Ra} \times \%$ gluconeogenesis), and the rate of glycogenolysis was calculated as the glucose production rate minus the rate of gluconeogenesis. Due to difficulties with the deuterated water supply at NIH CC, rates of gluconeogenesis were not measured in 2 White women.

A marker of SCD-1 ${ }_{16}$ activity is an indicator of hepatic de novo lipogenesis and was calculated as the plasma ratio of palmitoleic to palmitic acid as previously described (59).

The hepatic insulin sensitivity index $\left(\mathrm{HISI}_{\mathrm{GPR}}\right.$ ) was calculated in the fasting state: $1,000 /$ [glucose production $\left(\mathrm{mg} \cdot \mathrm{kg}_{\mathrm{LBM}}{ }^{-1} \cdot \mathrm{min}^{-1}\right) \times$ plasma insulin concentration $\left.(\mu \mathrm{U} / \mathrm{ml})\right](60)$. The adipose tissue insulin sensitivity index was calculated in the fasting state using the following formula: glycerol $\mathrm{Ra}\left(\mathrm{mg} \cdot \mathrm{kg}_{\mathrm{LBM}}{ }^{-1} \cdot \mathrm{min}^{-1}\right)$ $\times$ plasma insulin concentration $(\mu \mathrm{U} / \mathrm{ml})(61)$. The whole-body $\mathrm{S}_{\mathrm{I}}$, during the unlabeled IM-FSIGT, was calculated by the minimal model (MinMOD Millenium v.6.02) (51). $\mathrm{S}_{\mathrm{I}}$ results were not available in 3 women (2 Black and 1 White) because of hypoglycemia $(n=1)$, hemolyzed samples $(n=1)$, and difficulties with intravenous access $(n=1)$. The AIRg was calculated as the area under the insulin curve above basal between 0 and 10 minutes, and the DI was calculated as $\mathrm{S}_{\mathrm{I}} \times \mathrm{AIRg}$.

Statistics. Data are presented as mean \pm SEM, unless otherwise stated. The primary study outcome was the rate of glucose production between Black and White women. Secondary outcome analyses were measurements of fractional and absolute gluconeogenesis, $\mathrm{HISI}_{\mathrm{GPR}}$, and whole-body $\left(\mathrm{S}_{\mathrm{I}}\right)$. Differences between groups were tested by 2 -tailed $t$ tests for continuous variables and $\chi^{2}$ tests for categorical variables. Nonparametric data (insulin, glucagon, triglycerides, AIRg, DI, hepatic fat, and adipose tissue insulin sensitivity index) were natural log-transformed ( $\mathrm{Ln}$ ) prior to analyses. Pearson correlation coefficients (r) were used to compare continuous variables. A linear regression model was used to determine the relationship of $\mathrm{S}_{\mathrm{I}}$ with $\mathrm{HISI}_{\mathrm{GPR}}$, with race and race $\times \mathrm{S}_{\mathrm{I}}$ as covariates. All analyses were performed using STATA v. 15.0. A $P$ value of less than or equal to 0.05 was considered statistically significant.

Study approval. The protocol was approved by the NIDDK NIH Institutional Review Board (Clinicaltrials.gov NCT01809288). All participants provided signed written informed consent prior to participation.

\section{Author contributions}

STC designed the study, collected the data, conducted the analysis, and wrote the manuscript. MWH and AES contributed to study design and revised and edited the manuscript. AMG, PJW, HMG, LSM, CWD, MGDLC, AUO, ABC, SC, HC, and KK contributed to data collection and revised and edited the manuscript.

\section{Acknowledgments}

We would like to thank the volunteers, whose participation made this study possible. We gratefully acknowledge Pine Pharmaceuticals LLC for the compounding of the stable isotope intravenous infusion and Mary Walter (Core Laboratory, NIDDK NIH) and Susan Sharma (Children's Nutrition Research Center, Baylor College of Medicine) for assisting with sample analyses. STC, AMG, PJW, HMG, ABC, CWD, AES, and LSM are supported by the Intramural Research Program of the NIH, NIDDK. MWH and SC were supported by the US Department of Agriculture Current Research Information System (3092-51000-059-00D). The contents of this publication do not necessarily reflect the views or policies of the US Department of Agriculture or the Department of Health and Human Services, nor does mention of trade names, commercial products, or organizations imply endorsement from the US government.

Address correspondence to: Stephanie T. Chung, National Institute of Diabetes and Digestive and Kidney Diseases, NIH, 10 Center Drive Building 10-CRC, RM 5-3761, Bethesda, Maryland 20892-1612, USA. Phone: 301.402.2122; Email: chungst@niddk.nih.gov.

1. Ogurtsova K, et al. IDF Diabetes Atlas: Global estimates for the prevalence of diabetes for 2015 and 2040. Diabetes Res Clin Pract. 2017;128:40-50

2. Benjamin EJ, et al. Heart Disease and Stroke Statistics-2018 Update: A Report From the American Heart Association. Circulation. 2018;137(12):e67-e492.

3. Utumatwishima JN, Chung ST, Bentley AR, Udahogora M, Sumner AE. Reversing the tide - diagnosis and prevention of T2DM in populations of African descent. Nat Rev Endocrinol. 2018;14(1):45-56.

4. Chung ST, Sumner AE. Diabetes: T2DM risk prediction in populations of African descent. Nat Rev Endocrinol. 2016;12(3):131-132.

5. Price AJ, et al. Prevalence of obesity, hypertension, and diabetes, and cascade of care in sub-Saharan Africa: a cross-sectional, population-based study in rural and urban Malawi. Lancet Diabetes Endocrinol. 2018;6(3):208-222. 
6. Bansal N. Prediabetes diagnosis and treatment: A review. World J Diabetes. 2015;6(2):296-303.

7. American Diabetes Association. 2. Classification and Diagnosis of Diabetes. Diabetes Care. 2017;40(Suppl 1):S11-S24.

8. Bullard KM, et al. Secular changes in U.S. Prediabetes prevalence defined by hemoglobin A1c and fasting plasma glucose: National Health and Nutrition Examination Surveys, 1999-2010. Diabetes Care. 2013;36(8):2286-2293.

9. Caspersen CJ, Thomas GD, Beckles GL, Bullard KM. Secular changes in prediabetes indicators among older-adult Americans, 1999-2010. Am J Prev Med. 2015;48(3):253-263.

10. Davidson MB, Pan D. Epidemiological ramifications of diagnosing diabetes with HbA1c levels. J Diabetes Complicat. 2014;28(4):464-469

11. Levitt NS, et al. Application of the new ADA criteria for the diagnosis of diabetes to population studies in sub-Saharan Africa American diabetes association. Diabet Med. 2000;17(5):381-385

12. Ferguson TS, Tulloch-Reid MK, Wilks RJ. The epidemiology of diabetes mellitus in Jamaica and the Caribbean: a historical review. West Indian Med J. 2010;59(3):259-264.

13. Sumner AE, et al. Detection of abnormal glucose tolerance in Africans is improved by combining A1C with fasting glucose: the Africans in America Study. Diabetes Care. 2015;38(2):213-219.

14. Guerrero R, Vega GL, Grundy SM, Browning JD. Ethnic differences in hepatic steatosis: an insulin resistance paradox? Hepatol ogy. 2009;49(3):791-801.

15. Sumner AE, et al. Waist circumference, BMI, and visceral adipose tissue in white women and women of African descent. Obesity (Silver Spring). 2011;19(3):671-674.

16. Micklesfield LK, et al. Dual-energy X-ray absorptiometry and anthropometric estimates of visceral fat in Black and White South African Women. Obesity (Silver Spring). 2010;18(3):619-624.

17. der Merwe MT, et al. Free fatty acids and insulin levels--relationship to leptin levels and body composition in various patient groups from South Africa. Int J Obes Relat Metab Disord. 1999;23(9):909-917.

18. Eastwood SV, et al. Ethnic differences in associations between fat deposition and incident diabetes and underlying mechanisms: the SABRE study. Obesity (Silver Spring). 2015;23(3):699-706.

19. Knight MG, et al. The TG/HDL-C ratio does not predict insulin resistance in overweight women of African descent: a study of South African, African American and West African women. Ethn Dis. 2011;21(4):490-494.

20. Reuven Y, Dreiher J, Shvartzman P. The prevalence of diabetes, hypertension and obesity among immigrants from East Africa and the former Soviet Union: a retrospective comparative 30-year cohort study. Cardiovasc Diabetol. 2016;15:74.

21. Lacy ME, et al. Racial differences in the performance of existing risk prediction models for incident type 2 diabetes: The CARDIA Study. Diabetes Care. 2016;39(2):285-291.

22. Osei K, Cottrell DA, Harris B. Differences in basal and poststimulation glucose homeostasis in nondiabetic first degree relatives of black and white patients with type 2 diabetes mellitus. J Clin Endocrinol Metab. 1992;75(1):82-86.

23. Ellis AC, Alvarez JA, Granger WM, Ovalle F, Gower BA. Ethnic differences in glucose disposal, hepatic insulin sensitivity, and endogenous glucose production among African American and European American women. Metab Clin Exp. 2012;61(5):634-640.

24. DeLany JP, et al. Racial differences in peripheral insulin sensitivity and mitochondrial capacity in the absence of obesity. $J$ Clin Endocrinol Metab. 2014;99(11):4307-4314.

25. Goedecke JH, et al. Ethnic differences in hepatic and systemic insulin sensitivity and their associated determinants in obese black and white South African women. Diabetologia. 2015;58(11):2647-2652.

26. Chung ST, Chacko SK, Sunehag AL, Haymond MW. Measurements of gluconeogenesis and glycogenolysis: A Methodological Review. Diabetes. 2015;64(12):3996-4010.

27. Edgerton DS, et al. Small increases in insulin inhibit hepatic glucose production solely caused by an effect on glycogen metabolism. Diabetes. 2001;50(8):1872-1882.

28. Basu R, et al. Pathogenesis of prediabetes: role of the liver in isolated fasting hyperglycemia and combined fasting and postprandial hyperglycemia. J Clin Endocrinol Metab. 2013;98(3):E409-E417.

29. Chung ST, Hsia DS, Chacko SK, Rodriguez LM, Haymond MW. Increased gluconeogenesis in youth with newly diagnosed type 2 diabetes. Diabetologia. 2015;58(3):596-603.

30. Bock G, et al. Pathogenesis of pre-diabetes: mechanisms of fasting and postprandial hyperglycemia in people with impaired fasting glucose and/or impaired glucose tolerance. Diabetes. 2006;55(12):3536-3549.

31. Jiang X, Srinivasan SR, Radhakrishnamurthy B, Dalferes ER, Berenson GS. Racial (black-white) differences in insulin secretion and clearance in adolescents: the Bogalusa heart study. Pediatrics. 1996;97(3):357-360.

32. Osei K, Schuster DP. Metabolic characteristics of African descendants: a comparative study of African-Americans and Ghanaian immigrants using minimal model analysis. Diabetologia. 1995;38(9):1103-1109.

33. Osei K, Schuster DP, Owusu SK, Amoah AG. Race and ethnicity determine serum insulin and C-peptide concentrations and hepatic insulin extraction and insulin clearance: comparative studies of three populations of West African ancestry and white Americans. Metab Clin Exp. 1997;46(1):53-58.

34. Lee CC, et al. Insulin clearance and the incidence of type 2 diabetes in Hispanics and African Americans: the IRAS Family Study. Diabetes Care. 2013;36(4):901-907.

35. Polidori DC, Bergman RN, Chung ST, Sumner AE. Hepatic and extrahepatic insulin clearance are differentially regulated: results from a novel model-based analysis of intravenous glucose tolerance data. Diabetes. 2016;65(6):1556-1564.

36. Piccinini F, Polidori DC, Gower BA, Bergman RN. Hepatic but not extrahepatic insulin clearance is lower in African American than in European American women. Diabetes. 2017;66(10):2564-2570.

37. Brandimarti $\mathrm{P}$, et al. Cafeteria diet inhibits insulin clearance by reduced insulin-degrading enzyme expression and mRNA splicing. J Endocrinol. 2013;219(2):173-182.

38. Menke A, Rust KF, Savage PJ, Cowie CC. Hemoglobin A1c, fasting plasma glucose, and 2-hour plasma glucose distributions in U.S. population subgroups: NHANES 2005-2010. Ann Epidemiol. 2014;24(2):83-89.

39. Atiase Y, et al. A comparison of indices of glucose metabolism in five black populations: data from modeling the epidemiologic transition study (METS). BMC Public Health. 2015;15:895.

40. Adkins A, et al. Higher insulin concentrations are required to suppress gluconeogenesis than glycogenolysis in nondiabetic 
humans. Diabetes. 2003;52(9):2213-2220.

41. Staehr P, Hother-Nielsen O, Landau BR, Chandramouli V, Holst JJ, Beck-Nielsen H. Effects of free fatty acids per se on glucose production, gluconeogenesis, and glycogenolysis. Diabetes. 2003;52(2):260-267.

42. Boden G. Effects of free fatty acids (FFA) on glucose metabolism: significance for insulin resistance and type 2 diabetes. Exp Clin Endocrinol Diabetes. 2003;111(3):121-124.

43. Arslanian SA, Saad R, Lewy V, Danadian K, Janosky J. Hyperinsulinemia in African-American children: decreased insulin clearance and increased insulin secretion and its relationship to insulin sensitivity. Diabetes. 2002;51(10):3014-3019.

44. Hannon TS, Bacha F, Lin Y, Arslanian SA. Hyperinsulinemia in African-American adolescents compared with their American white peers despite similar insulin sensitivity: a reflection of upregulated beta-cell function? Diabetes Care. 2008;31(7):1445-1447.

45. Rasouli N, Spencer HJ, Rashidi AA, Elbein SC. Impact of family history of diabetes and ethnicity on -cell function in obese, glucose-tolerant individuals. J Clin Endocrinol Metab. 2007;92(12):4656-4663.

46. Haffner SM, et al. Increased insulin resistance and insulin secretion in nondiabetic African-Americans and Hispanics compared with non-Hispanic whites. The Insulin Resistance Atherosclerosis Study. Diabetes. 1996;45(6):742-748.

47. Chow CC, et al. Higher acute insulin response to glucose may determine greater free fatty acid clearance in African-American women. J Clin Endocrinol Metab. 2011;96(8):2456-2463.

48. Albu JB, Curi M, Shur M, Murphy L, Matthews DE, Pi-Sunyer FX. Systemic resistance to the antilipolytic effect of insulin in black and white women with visceral obesity. Am J Physiol. 1999;277(3 Pt 1):E551-E560.

49. Mifflin MD, St Jeor ST, Hill LA, Scott BJ, Daugherty SA, Koh YO. A new predictive equation for resting energy expenditure in healthy individuals. Am J Clin Nutr. 1990;51(2):241-247.

50. Boston RC, Stefanovski D, Moate PJ, Sumner AE, Watanabe RM, Bergman RN. MINMOD Millennium: a computer program to calculate glucose effectiveness and insulin sensitivity from the frequently sampled intravenous glucose tolerance test. Diabetes Technol Ther. 2003;5(6):1003-1015.

51. Sumner AE, et al. Validity of the reduced-sample insulin modified frequently-sampled intravenous glucose tolerance test using the nonlinear regression approach. Metab Clin Exp. 2009;58(2):220-225.

52. Ferrannini E. The theoretical bases of indirect calorimetry: a review. Metab Clin Exp. 1988;37(3):287-301.

53. Livesey G, Elia M. Estimation of energy expenditure, net carbohydrate utilization, and net fat oxidation and synthesis by indirect calorimetry: evaluation of errors with special reference to the detailed composition of fuels. Am J Clin Nutr. 1988;47(4):608-628.

54. Ouwerkerk R, Pettigrew RI, Gharib AM. Liver metabolite concentrations measured with 1H MR spectroscopy. Radiology. 2012;265(2):565-575.

55. Podszun MC, Rolt A, Garaffo HM, Walter PJ. Vitamin E (RRR-alpha-tocopherol) decreases hepatic de novo lipogenesis. Free Radic Biol Med. 2017;112:30-31.

56. Chacko SK, Sunehag AL, Sharma S, Sauer PJ, Haymond MW. Measurement of gluconeogenesis using glucose fragments and mass spectrometry after ingestion of deuterium oxide. J Appl Physiol. 2008;104(4):944-951.

57. Walter PJ, Garraffo HM, Chung S, Brown RJ. Clinical tracers: A simple, rapid and simultaneous LC-MS method to measure stable isotope enrichment of glucose, glycerol and palmitic acid. Poster presented at: the 65th ASMS Conference on Mass Spectrometry and Allied Topics; June 4-8, 2017; Indianapolis, Indiana, USA. https://www.asms.org/docs/default-source/asms2017/65thasms-program_full_web_v2.pdf?sfvrsn=0.

58. Sunehag AL, et al. Glucose production, gluconeogenesis, and insulin sensitivity in children and adolescents: an evaluation of their reproducibility. Pediatr Res. 2001;50(1):115-123.

59. Lee JJ, et al. Palmitoleic acid is elevated in fatty liver disease and reflects hepatic lipogenesis. Am J Clin Nutr. 2015;101(1):34-43.

60. Matsuda M, DeFronzo RA. Insulin sensitivity indices obtained from oral glucose tolerance testing: comparison with the euglycemic insulin clamp. Diabetes Care. 1999;22(9):1462-1470

61. Kim JY, Nasr A, Tfayli H, Bacha F, Michaliszyn SF, Arslanian S. Increased lipolysis, diminished adipose tissue insulin sensitivity, and impaired $\beta$-cell function relative to adipose tissue insulin sensitivity in obese youth with impaired glucose tolerance. Diabetes. 2017;66(12):3085-3090. 